\title{
Crossing Numbers of Meshes
}

\author{
Farhad Shahrokhi ${ }^{1}$, Ondrej Sýkora ${ }^{2, \star}$, László A. Székely ${ }^{3}$, Imrich Vrt'o ${ }^{2}$ \\ 1 Department of Computer Science, University of North Texas \\ P.O.Box 13886, Denton, TX, USA \\ 2 Institute for Informatics, Slovak Academy of Sciences \\ P.O.Box 56, 84000 Bratislava, Slovak Republic \\ 3 Department of Computer Science, Eötvös University \\ Múzeum krt. 6-8, 1088 Budapest, Hungary
}

\begin{abstract}
We prove that the crossing number of the cartesian product of 2 cycles, $C_{m} \times C_{n}, m \leq n$, is of order $\Omega(m n)$, improving the best known lower bound. In particular we show that the crossing number of $C_{m} \times C_{n}$ is at least $m n / 90$, and for $n=m, m+1$ we reduce the constant 90 to 6 . This partially answers a 20-years old question of Harary, Kainen and Schwenk [3] who gave the lower bound $m$ and the upper bound $(m-2) n$ and conjectured that the upper bound is the actual value of the crossing number for $C_{m} \times C_{n}$. Moreover, we extend this result to $k \geq 3$ cycles and paths, and obtain such lower and upper bounds on the crossing numbers of the corresponding meshes, which differ by a small constant only.
\end{abstract}

\section{Introduction}

The crossing number of a graph $G$, denoted by $\operatorname{cr}(G)$, is the minimum number of crossings of its edges over all drawings of $G$ in the plane, such that no more than two edges intersect in any point and no edge passes through a vertex. Computing $\operatorname{cr}(G)$ is NP-hard and there have been only few results concerning the exact value of crossing number for very special and restricted classes of graphs. Besides Kleitman's exact result [6] on the crossing number of $K_{m, n}$, for $m \leq 6$, most effort has been devoted to crossing numbers of some cartesian product graphs $[4,7,8,22]$. For a detailed exposition of the crossing number problem see our survey [19].

For $G_{1}=\left(V_{1}, V_{2}\right)$ and $G_{2}=\left(V_{2}, E_{2}\right)$, let $G_{1} \times G_{2}$ denote the cartesian product of $G_{1}$ and $G_{2}$. Thus $G_{1} \times G_{2}$ is a graph with the vertex set $V_{1} \times V_{2}$ in which $(i, j)(r, s)$ are adjacent iff either $i=r$ and $j s \in E_{2}$ or $j=s$ and $i r \in E_{1}$. Let $P_{n}$ and $C_{n}$ denote the $n$-vertex path and the $n$-vertex cycle, respectively. For $2 \leq n_{1} \leq n_{2} \leq \ldots \leq n_{k}$, let $M_{k}=\prod_{i=1}^{k} P_{n_{i}}$ and $T M_{k}=\prod_{i=1}^{k} C_{n_{i}}$. We will call $M_{k}$ and $T M_{k}$ the $k$-dimensional mesh and the $k$-dimensional toroidal mesh, respectively.

* Research of the 2nd and the 4th author was partially supported by grant No. 2/1138/94 of Slovak Grant Agency and Alexander von Humboldt Foundation. 
Clearly, $M_{2}$ is planar and has crossing number 0 , but estimating the crossing number of $T M_{2}$ has been an open problem. Harary et al. [3] provided a simple drawing of $C_{m} \times C_{n}, m \leq n$ with $(m-2) n$ crossings. They also derived a weak lower bound $m$ on $\operatorname{cr}\left(C_{m} \times C_{n}\right)$ and conjectured that $\operatorname{cr}\left(C_{m} \times C_{n}\right)=(m-2) n$, for $3 \leq m \leq n$. Beineke and Ringeisen $[1,16]$ proved the conjecture for $m \leq 4$. Richter and Thomassen [15] proved the conjecture for $m=n=5$. Finally, very recently Klešc [9] and Richter and Stobert [14] announced their proof for $m=5$ and arbitrary $n \geq 5$. It is instructive to mention that all existing standard methods for estimating lower bounds on the crossing number fail to give good lower bounds for $\operatorname{cr}\left(C_{m} \times C_{n}\right)$. In particular two very powerful methods developed by VLSI community [11] - the bisection method and the embedding method give a weak lower bound for $\operatorname{cr}\left(C_{m} \times C_{n}\right)$. We suspect that the reason is that $C_{m} \times C_{n}$ has genus 1 and very much resembles the planar 2-dimensional mesh.

In this paper we take a major step to prove the conjecture and show that for $6 \leq m \leq n, \operatorname{cr}\left(C_{m} \times C_{n}\right) \geq m n / 90$. For $n=m, m+1$ we improve the constant 90 to 6 . It is worth mentioning that the method used here to prove our main result employs Dilworth' chain decomposition theorem which was shown to be very effective when dealing with problems in combinatorial geometry $[10,13]$.

Moreover for $k \geq 3$, we derive upper and lower bounds within a constant multiplicative factor for the crossing number of $M_{k}$ and $T M_{k}$. We indicate that, since bisection width of $M_{k}$ and $T M_{k}$ are known, see for instance [12], one can use the relationship between the crossing number and the bisection and derive lower bounds for $\operatorname{cr}\left(M_{k}\right)$ and $\operatorname{cr}\left(T M_{k}\right), k \geq 3$ which are of the same order magnitude as the lower bounds we have derived here. Nevertheless, such an approach does not provide constants, whereas, our method identifies relatively large constants associated with our lower bounds. Moreover, for $k \geq 3$ we have been able to provide (new) drawings of $M_{k}$ and $T M_{k}$, with number of crossings which are within relatively small constant factors from the lower bounds, under reasonable conditions.

\section{Crossing Number of $C_{m} \times C_{n}$}

One finds in $C_{m} \times C_{n}$ in a natural way $n$ vertex disjoint row cycles and $m$ vertex disjoint column cycles. We will call them $r$-cycles and c-cycles. Deleting all edges of any r-cycle (c-cycle) yields a graph which is a subdivision of $C_{m} \times C_{n-1}$ (of $\left.C_{m-1} \times C_{n}\right)$ and therefore has a crossing number less than or equal to that of $C_{m} \times C_{n}$. It immediately follows, that $\operatorname{cr}\left(C_{m} \times C_{n}\right)$ is monotone nondecreasing in both parameters. We will use this fact implicitly throughout our proof many times.

Theorem 2.1 For $6 \leq m \leq n$,

$$
\operatorname{cr}\left(C_{m} \times C_{n}\right) \geq \frac{m n}{90} .
$$


Proof. If $6 \leq m \leq n \leq 180$ then by monotonicity and a result of Beineke and Ringeisen [1]: $\operatorname{cr}\left(\bar{C}_{4} \times \bar{C}_{n}\right)=2 n$ we have

$$
\operatorname{cr}\left(C_{m} \times C_{n}\right) \geq \operatorname{cr}\left(C_{4} \times C_{n}\right)=2 n \geq \frac{m n}{90} .
$$

Assume $n \geq 181$. For a closed curve $C$ in the plane $R^{2}$, define its body $B(C)$ as the closure of the union of the bounded components of $R^{2} \backslash C$. See Fig. 1. Define the exterior of $C$ as the complement of $B(C)$ (the white part of Fig. 1).

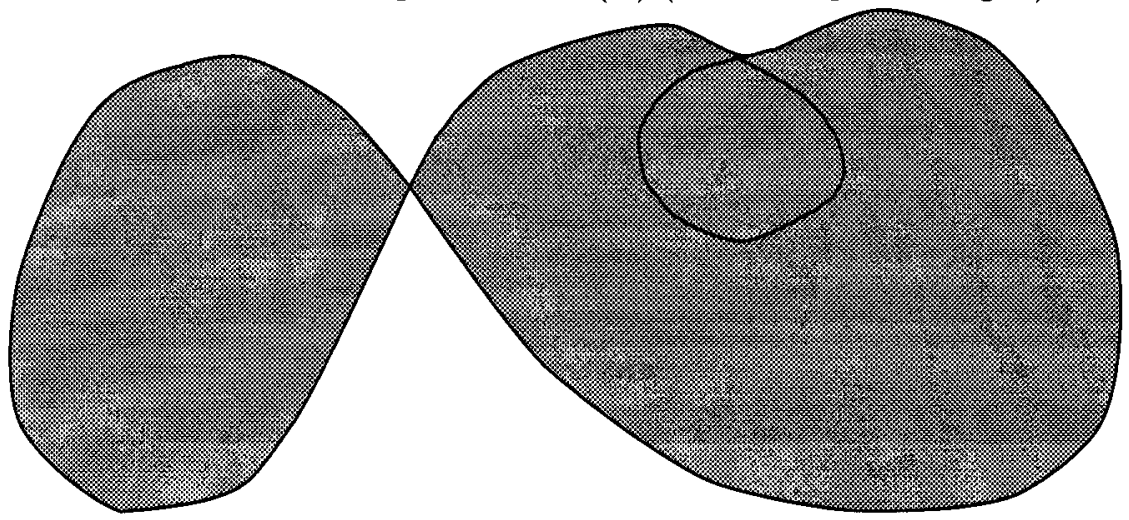

Fig. 1 : The body of a closed curve

Let us be given a drawing $D$ of $C_{m} \times C_{n}$ in the plane. We may assume without loss of generality that (1) crossing edges really cross, i.e. no touching situation occurs, (2) the number of crossings in $D$ is finite. Define a partial order on the set of its r-cycles by $C<Z$ if $C$ lies in one of the bounded connected components of $R^{2} \backslash Z$. Dilworth' Theorem [2] p. 62 applies to this poset. If $k$ is the size of the largest antichain, then the poset may be decomposed into chains of length $a_{1}, a_{2}, \ldots, a_{k}$ such that $a_{1}+a_{2}+\ldots+a_{k}=n$. In such a chain each member contains the next member of the chain in one of the bounded connected components of the plane that it defines. Each c-cycle has exactly one vertex on every member cycle in the $i$-th chain. Hence, by Jordan's Curve Theorem, every c-cycle has at least $a_{i}-2$ crossings with the $\mathrm{r}$-cycles of the $i$-th chain. Therefore, $\operatorname{cr}(D) \geq m\left(a_{1}+a_{2}+\ldots+a_{k}-2 k\right)=m(n-2 k) \geq m n / 50$, if $k<49 n / 100$.

Assume that $k \geq 49 n / 100$. We have an antichain of size at least $49 n / 100$, i.e. at least $49 n / 100 \mathrm{r}$-cycles such that any two cross or they lie in the exterior of each other. Ignoring the edges of the other r-cycles, we consider our drawing as

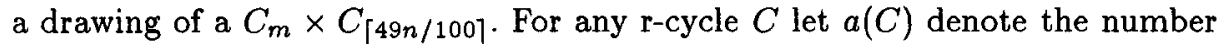
of c-cycles whose bodies lie in the body of $C$. Observe that for two r-cycles $C, Z$ taken from an antichain, $a(C)>0$ implies 2 crossing of $C$ and $Z$, since every c-cycle was supposed to have a common vertex with every $r$-cycle and if two r-cycles cross then they must cross at least twice. Either we have at least $(1 / 24)(49 n / 100) \mathrm{r}$-cycles $C$ with $a(C)>0$, and the total number of crossings is $2(49 n / 2400) \cdot(49 n / 100-1)>m n / 90$ and we are at home, or we have at least $(23 / 24)(49 n / 100)$ r-cycles $C$ with $a(C)=0$. Reduce our drawing further to the drawing of the corresponding $C_{m} \times C_{\lceil 1127 n / 2400]}$. 
Now every c-cycle either crosses an r-cycle or is in the exterior of it. Either we have at least $(1 / 3)(1127 n / 2400)$ r-cycles, such that each of them is crossed by the c-cycles at least $m / 14$ times, or we have at least $(2 / 3)(1127 n / 2400) \mathrm{r}$ cycles, such that the c-cycles cross them at most $m / 14$ times. In the first case $\operatorname{cr}(D) \geq m n / 90$, and the Theorem holds. In the second case reduce our interest to a drawing $D$ of $G=C_{m} \times C_{\lceil 1127 n / 3600\rceil}$ so that for every r-cycle $C, a(C)=0$ and at most $m / 14 \mathrm{c}$-cycles cross $C$. Let $\operatorname{cr}(D)$ denote the number of crossings in $D$. The proof is completed employing the following claim.

Claim: $D$ can be extended to a drawing $D^{\prime}$ of a graph $G^{\prime}$ with $\operatorname{cr}\left(G^{\prime}\right) \geq m n / 15$ and $\operatorname{cr}\left(D^{\prime}\right) \leq 6 \mathrm{cr}(D)$.

Proof of the Claim. We construct $D^{\prime}$ by adding edges to each r-cycle $C$. In particular we add at least $3 \mathrm{~m} / 14$ new edges to each r-cycle $C$.

Let $S$ be the set of all c-cycles that cross $C$. By deleting all edges of $C$ that are crossed by c-cycles from $S$, we divide $C$ to at most $\lfloor m / 14\rfloor$ vertex-disjoint paths. On these paths, there are at most $\lfloor m / 14\rfloor$ vertices, in which c-cycles from $S$ have a vertex in common with $C$, since each $\mathbf{r}$-cycle and each c-cycle have exactly one vertex in common. These vertices divide the $[m / 14\rfloor$ vertex disjoint paths into at most $2\lfloor m / 14\rfloor$ edge disjoint paths of lengths $d_{1}, d_{2}, d_{3}, \ldots, d_{2\lfloor m / 14\rfloor}$, where $\sum_{i=1}^{2\lfloor m / 14\rfloor} d_{i} \geq m-\lfloor m / 14\rfloor$. To each path of length $d_{i}$, we can add at least $\left\lfloor d_{i} / 3\right\rfloor$ new edges so that

(i) in each path above we join vertices of distance 3 in a greedy way, such that the new "long edges" do not overlap

(ii) the new edges are drawn very close to $C$ and inside $B(C)$, as far as it is possible

(iii) the new edges do not cross each other unless the corresponding part of the drawing of $C$ is self-intersecting

(iv) the new edges do not cross c-cycles.

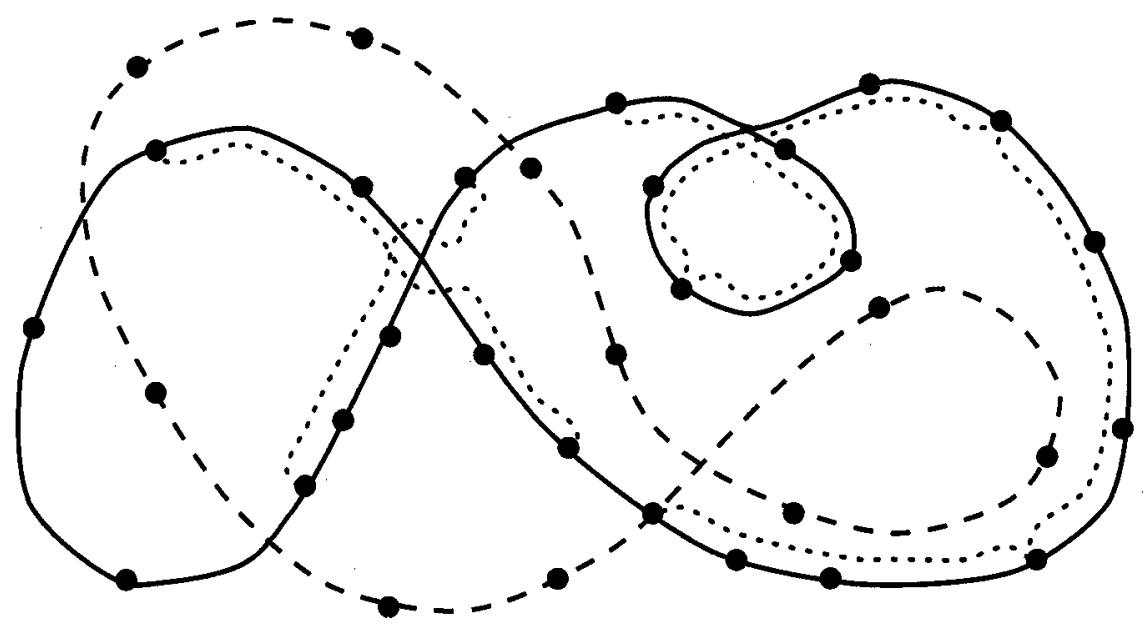

Fig. 2 
Fig. 2 shows details of a drawing of $C_{23} \times C_{11}$ with one r-cycle (solid line) and one c-cycle (dashed line). The new edges are drawn by dotted lines. Hence the total number of added edges is

$$
\sum_{i=1}^{2\left\lfloor\frac{m}{14}\right\rfloor}\left\lfloor\frac{d_{i}}{3}\right\rfloor \geq \sum_{i=1}^{2\left\lfloor\frac{m}{14}\right\rfloor} \frac{d_{i}-2}{3} \geq \frac{1}{3}\left(m-\left\lfloor\frac{m}{14}\right\rfloor-4\left\lfloor\frac{m}{14}\right\rfloor\right) \geq \frac{3 m}{14} .
$$

The drawing $D^{\prime}$ obtained in this way corresponds to a graph $G^{\prime}$ which has $m\lceil 1127 n / 3600\rceil$ vertices, at least $2 m\lceil 1127 n / 3600\rceil+161 m n / 2400$ edges, and of girth 4 . Due to the construction $\operatorname{cr}\left(D^{\prime}\right) \leq 6 \operatorname{cr}(D)$, since a crossing of two distinct r-cycles in $D$ may result in at most 4 crossings in $D^{\prime}$, a self-crossing of an r-cycle in $D$ may result in 6 crossings in $D^{\prime}$ (see Fig. 2). Crossings of r-cycles and c-cycles do not multiply. Finally, Kainen's lower bound [5] applies to $G^{\prime}$ :

$$
\operatorname{cr}\left(G^{\prime}\right) \geq\left|E\left(G^{\prime}\right)\right|-\frac{\operatorname{girth}\left(G^{\prime}\right)}{\operatorname{girth}\left(G^{\prime}\right)-2}\left(\left|V\left(G^{\prime}\right)\right|-2\right) .
$$

This formula immediately finishes the proof.

In special cases, we can improve the lower bound to the following:

Theorem 2.2 For $3 \leq m \leq n$, where $n=m$ or $m+1$

$$
\operatorname{cr}\left(C_{m} \times C_{n}\right) \geq \frac{(m-2) n}{6} .
$$

Proof. We inductively show that if the claim holds for $\operatorname{cr}\left(C_{m} \times C_{m}\right)$, then it also holds for $\operatorname{cr}\left(C_{m} \times C_{m+1}\right)$, and use this to show that the claim holds for $\operatorname{cr}\left(C_{m+1} \times C_{m+1}\right)$. We call the crossing of two different r-cycles as rr-crossing. Similarly define the cc-crossing and the rc-crossing. The claim is true for $C_{m} \times$ $C_{m}$ when $m \leq 10$, as $\operatorname{cr}\left(C_{m} \times C_{m}\right)=(m-2) m$, for $m \leq 5,[1,16,15]$ and $\operatorname{cr}\left(C_{m} \times C_{m}\right) \geq \operatorname{cr}\left(C_{5} \times C_{5}\right)=15 \geq(m-2) m / 6$, for $m=6,7, \ldots, 10$. Let the claim hold for $C_{m} \times C_{m}, m \geq 10$. We first show it holds for $C_{m} \times C_{m+1}$. Consider any drawing of $C_{m} \times C_{m+1}$. Suppose that there exists an r-cycle in $C_{m} \times C_{m+1}$ containing at least $m / 6$ crossings. Deleting the edges of this $\mathbf{r}$-cycle we get a subdivision of $C_{m} \times C_{m}$. Hence

$$
\operatorname{cr}\left(C_{m} \times C_{m+1}\right) \geq \operatorname{cr}\left(C_{m} \times C_{m}\right)+\frac{m}{6} \geq \frac{(m-2) m}{6}+\frac{m}{6} \geq \frac{(m-2)(m+1)}{6} .
$$

Suppose that each r-cycle in $C_{m} \times C_{m+1}$ contains at most $\lfloor m / 6\rfloor$ crossings. We may assume that there exist 3 distinct $r$-cycles, so that no two of them have a crossing. Otherwise each triplet of r-cycles would determine at least 2 rr-crossings and a simple counting argument shows that

$$
\operatorname{cr}\left(C_{m} \times C_{m+1}\right) \geq \frac{2\left(\begin{array}{c}
m+1 \\
3
\end{array}\right)}{m-1}>\frac{(m-2)(m+1)}{6} .
$$

Consider the 3 distinct r-cycles. Since for each of them, there exist at least $m-\lfloor m / 6\rfloor$ c-cycles, which do not cross it, there are at least $m-3\lfloor m / 6\rfloor$ ccycles, none of them crosses any of the 3 distinct r-cycles. Now every triplet 
of these $m-3\lfloor m / 6\rfloor$ c-cycles together with the 3 distinct r-cycles determine a subdivision of $C_{3} \times C_{3}$. If the subdivision contains a selfcrossing of a cycle we can redraw it without the selfcrossing and without producing a new crossing. Hence we may assume that the subdivision is without selfcrossings. It has 3 cc-crossings, since $\operatorname{cr}\left(C_{3} \times C_{3}\right)=3$, but it must contain an even number of cccrossings, since c-cycles are vertex disjoint and if they cross, they must cross an even number of times. Thus, this subdivision contains at least $4 \mathrm{cc}$-crossing. Further, a counting argument shows that,

$$
\operatorname{cr}\left(C_{m} \times C_{m+1}\right) \geq \frac{4\left(\begin{array}{c}
m-3\lfloor m / 6\rfloor \\
3
\end{array}\right)}{m-3\lfloor m / 6\rfloor-2} \geq \frac{(m-2)(m+1)}{6} .
$$

We may conclude that if the claim holds for $C_{m} \times C_{m}$ it also holds for $C_{m} \times C_{m+1}$. Now we use this fact to prove a lower bound for $C_{m+1} \times C_{m+1}$. Suppose that there exists an r-cycle in $C_{m+1} \times C_{m+1}$ containing at least $(m+1) / 6$ crossings. Deleting the edges of the r-cycle we get a subdivision of $C_{m+1} \times C_{m}$. Hence

$$
\begin{aligned}
c r\left(C_{m+1} \times C_{m+1}\right) & \geq c r\left(C_{m+1} \times C_{m}\right)+\frac{m+1}{6} \geq \frac{(m-2)(m+1)}{6}+\frac{m+1}{6} \\
& \geq \frac{(m-1)(m+1)}{6} .
\end{aligned}
$$

Suppose that each r-cycle in $C_{m+1} \times C_{m+1}$ contains at most $(m+1) / 6$ crossings. The rest of the proof is the same as the proof of the lower bound for $C_{m} \times C_{m+1}$.

\section{Crossing Number of $M_{k}$ and $T M_{k}$}

It is straightforward to show, using Theorem 2.1 that, for $k \geq 3, \operatorname{cr}\left(T M_{k}\right)=$ $\Omega\left(\Pi_{i=1}^{k} n_{i}\right)$ which is a weak lower bound in some cases. In this section we prove near-optimal lower and upper bounds on the crossing number of $T M_{k}$ and $M_{k}$, for $k \geq 3$. In particular, when $n_{i}=n, i=1,2, . ., k$, the upper and lower bounds differ by a multiplicative factor only. Let $G_{1}=\left(V_{1}, E_{1}\right)$ and $G_{2}=\left(V_{2}, E_{2}\right)$ be graphs such that $\left|V_{1}\right| \leq\left|V_{2}\right|$.

An embedding $\omega$ of $G_{1}$ in $G_{2}$ is a pair of injections $(\phi, \psi)$

$$
\left.\phi: V_{1} \rightarrow V_{2}, \quad \psi: E_{1} \rightarrow \text { all paths in } G_{2}\right\},
$$

such that if $u v \in E_{1}$ then $\psi(u v)$ is a path between $\phi(u)$ and $\phi(v)$. Define the congestion of $\omega$

$$
\mu_{\omega}=\max _{e \in E_{2}}\left\{\left|\left\{f \in E_{1}: e \in \psi(f)\right\}\right|\right\} .
$$

Leighton [11] invented a lower bound technique for crossing numbers, based on an embedding of the complete graph in the given graph. Several authors $[18,20,22]$ realized that the method can be generalized for arbitrary graphs in the following form: 
Lemma 3.1 Let $\omega$ be an embedding of $G_{1}=\left(V_{1}, E_{1}\right)$ into $G_{2}=\left(V_{2}, E_{2}\right)$, $\left|V_{1}\right| \leq\left|V_{2}\right|$. Let $D_{2}$ be a drawing of $G_{2}$ with $\operatorname{cr}\left(D_{2}\right)$ crossings, then there is a drawing $D_{1}$ of $G_{1}$ with $\operatorname{cr}\left(D_{1}\right)$ crossings so that

$$
\operatorname{cr}\left(D_{2}\right) \geq \frac{\operatorname{cr}\left(D_{1}\right)}{\mu_{\omega}^{2}}-\frac{\left|V_{2}\right| \Delta_{2}^{2}}{2}
$$

where $\Delta_{2}$ is the maximum degree of $G_{2}$.

For $2 \leq n_{1} \leq n_{2} \ldots . \leq n_{k}$, let $N_{i}=n_{1} n_{2} \ldots n_{i}$, for $i=1,2, \ldots, k$.

Theorem 3.1 For $k \geq 3$

$$
\begin{gathered}
\frac{N_{k-1}^{2}}{5}-\frac{\left(5 k^{2}+8\right) N_{k}}{2} \leq \operatorname{cr}\left(M_{k}\right) \leq 4 N_{k-2} N_{k}, \\
\frac{4 N_{k-1}^{2}}{5}-2\left(k^{2}+2\right) N_{k} \leq \operatorname{cr}\left(T M_{k}\right) \leq 16 N_{k-2} N_{k}+8 k^{2} N_{k} .
\end{gathered}
$$

Proof. We first prove the lower bound for $\operatorname{cr}\left(T M_{k}\right)$ and construct an upper bound for $\operatorname{cr}\left(M_{k}\right)$. Set $G_{2}=T M_{k}$ and $G_{1}=2 K_{N_{k}}$, where $2 K_{N_{k}}$ denote the complete multigraph on $N_{k}$ vertices, obtained from $K_{N_{k}}$ by replacing every edge by two new edges. Shahrokhi and Székely [17] constructed an embedding $\omega$ of $G_{1}$ into $G_{2}$ with

$$
\mu_{\omega} \leq \frac{N_{k} n_{k}}{4} .
$$

Substituting this into Lemma 3.1, recalling from [21] the following formula:

$$
\operatorname{cr}\left(K_{N_{k}}\right) \geq \frac{N_{k}^{4}-7 N_{k}^{3}}{80}
$$

and noting that $\operatorname{cr}\left(2 K_{N_{k}}\right)=4 \operatorname{cr}\left(K_{N_{k}}\right)$ we get the claimed lower bound for $T M_{k}$.

Now we prove an upper bound for $\operatorname{cr}\left(M_{k}\right)$. We construct a recursive drawing $L_{k}$ for $M_{k}$ in which all vertices are placed along a straight line in the plane. For $M_{1}=P_{n_{1}}$ we place successively the vertices of $P_{n_{1}}$ on a line and obtain a drawing with no crossings. Assume that we have constructed a drawing $L_{k-1}$ of $M_{k-1}$ with $\operatorname{cr}\left(L_{k-1}\right)$ crossings. The drawing $L_{k}$ of $M_{k}=M_{k-1} \times P_{n_{k}}$ is constructed in the following way. Place $n_{k}$ copies of the drawings $L_{k-1}$ successively on a line such that 2 neighboring copies are symmetric according to a perpendicular line between the 2 copies. Join the corresponding vertices of the first and the second copy by edges drawn as half-circles above the line. Similarly, join the corresponding vertices of the second and the third copy by edges drawn as halfcircles below the line and continue in this fashion until a drawing $L_{k}$ of $M_{k}$ is obtained. Let us call the inserted edges the edges of the dimension $k$ and denote the number of crossings in $L_{k}$ by $\operatorname{cr}\left(L_{k}\right)$. Clearly

$$
\operatorname{cr}\left(L_{k}\right) \leq n_{k} \operatorname{cr}\left(L_{k-1}\right)+l_{k},
$$

where $l_{k}$ denotes the number of crossings of the edges of the $k$-th dimension with edges of $n_{k}$ copies of the drawing $L_{k-1}$, i.e. the number of crossings of edges of 
the $k$-th dimension with edges of smaller dimensions. A counting analysis shows that there are at most $N_{k} N_{i-1}$ crossings of the edges of the $k$-th dimension with edges of the $i$-th dimension, where $N_{0}=1$. Hence

$$
l_{k} \leq N_{k} \sum_{i=1}^{k-1} N_{i-1}
$$

and

$$
\operatorname{cr}\left(L_{k}\right) \leq n_{k} \operatorname{cr}\left(L_{k-1}\right)+N_{k} \sum_{i=0}^{k-2} N_{i}
$$

The solution is

$$
\begin{aligned}
\operatorname{cr}\left(L_{k}\right) & \leq N_{k} \sum_{i=0}^{k-2}(k-i-1) N_{i} \\
& \leq N_{k} N_{k-2}\left(1+\frac{2}{n_{k-2}}+\frac{3}{n_{k-2} n_{k-3}}+\ldots+\frac{k-1}{n_{k-2} \ldots n_{1}}\right) \leq 4 N_{k} N_{k-2} .
\end{aligned}
$$

Finally, we use Lemma 3.1 with $G_{1}=T M_{k}$ and $G_{2}=M_{k}$ and note that there is an embedding $\omega$ of $G_{1}$ into $G_{2}$ with $\mu_{\omega}=2$. To get the upper bound for $\operatorname{cr}\left(T M_{k}\right)$, we take $D_{2}$ to be $L_{k}$, then $D_{1}$ is a desirable drawing of $T M_{k}$. To get the lower bound for $\operatorname{cr}\left(M_{k}\right)$, we substitute the term $\operatorname{cr}\left(D_{1}\right)$ in the Lemma, by our lower bound for $\operatorname{cr}\left(T M_{k}\right)$.

Corollary 3.1 If $n_{k}=O\left(n_{k-1}\right)$ then the bounds in Theorem 3.1 are optimal within a constant multiplicative factor and in addition, if $n_{1}=n_{2}=\ldots=n_{k}=n$ then

$$
\begin{gathered}
\frac{n^{2 k-2}}{5}-\frac{\left(5 k^{2}+8\right) n^{k}}{2} \leq \operatorname{cr}\left(M_{k}\right) \leq 4 n^{2 k-2}, \\
\frac{4 n^{2 k-2}}{5}-2\left(k^{2}+2\right) n^{k} \leq \operatorname{cr}\left(T M_{k}\right) \leq 16 n^{2 k-2}+8 k^{2} n^{k} .
\end{gathered}
$$

Corollary 3.2 Consider a general $k$-dimensional mesh $G M_{k}=\prod_{i=1}^{k} A_{n_{i}}$, where $A_{n_{i}}$ equals either $P_{n_{i}}$ or $C_{n_{i}}$. Then

$$
\operatorname{cr}\left(M_{k}\right) \leq \operatorname{cr}\left(G M_{k}\right) \leq \operatorname{cr}\left(T M_{k}\right) \text {. }
$$




\section{References}

1. L. W. Beineke, R. D. Ringeisen, On the crossing number of product of cycles and graphs of order four, J. Graph Theory 4 (1980), 145-155.

2. M. Hall, Jr., Combinatorial Theory, Blaisdell Publ. Co., Waltham, 1967.

3. F. Harary, P. C. Kainen, A. Schwenk, Toroidal graphs with arbitrary high crossing numbers, Nanta Mathematica 6 (1973), 58-67.

4. S. Jendrol', M. Śčerbová, On the crossing numbers of $S_{m} \times P_{n}$ and $S_{m} \times C_{n}$, Casopis pro Pestováni Matematiky 107 (1982), 225-230.

5. P. C. Kainen, A lower bound for crossing number of graphs with applications to $K_{n}, K_{p, q}$, and $Q(d), J$. Combinatorial Theory, Series $B 12$ (1972), 287-298.

6. D. J. Kleitman, The crossing number of $K_{5, n}, J$. Combinatorial Theory 9 (1970), 315-323.

7. M. Klešč, On the crossing number of the cartesian product of stars and paths or cycles, Mathematica Slovaca 41 (1991), 113-120.

8. M. Klešč, The crossing number of product of path and stars with 4-vertex graphs, J. Graph Theory 18 (1994), 605-614.

9. M. Klešč, On the crossing numbers of products of cycles, preprint.

10. D. Larman, J. Matoušek, J. Pach, J. Töröcsik, A Ramsey-type result for planar convex sets, to appear.

11. F. T. Leighton, Complexity Issues in VLSI, MIT Press, Cambridge, 1983.

12. F. T. Leighton, Introduction to Parallel Algorithms and Architectures: Arrays.Trees.Hypercubes, Morgan Kaufmann, San Mateo, 1992.

13. J. Pach, J. Töröcsik, Some geometric applications of Dilworth' theorem, Discrete Computational Geometry, 21(1994), 1-7.

14. R. B. Richter, I. Stobert, The crossing number of $C_{5} \times C_{n}$, preprint.

15. R. B. Richter, C. Thomassen, Intersection of curve systems and the crossing number of $C_{5} \times C_{5}$, Discrete and Computational Geometry 13 (1995), 149-159.

16. R. D. Ringeisen, L. W. Beineke, The crossing number of $C_{3} \times C_{n}, J$. Combinnatorial Theory, Series B 24 (1978), 134-144.

17. F. Shahrokhi, L. A. Székely, An algebraic approach to the uniform concurrent multicommodity flow problem: theory and applications, Technical Report CRPDC91-4, Department of Computer Science, University of North Texas, Denton, 1991.

18. F. Shahrokhi, L. A. Székely, Effective lower bounds for crossing number, bisection width and balanced vertex separators in terms of symmetry, in: Proc. 2-nd IPCO Conference, Pittsburgh, 1992, 102-113, also in Combinatorics, Probability and Computing 3 (1994), 523-543.

19. F. Shahrokhi, L. A. Székely, I. Vrto, Crossing numbers of graphs, lower bound techniques and algorithms: a survey, in: Proc. DIMACS Workshop on Graph Drawing'94, Lecture Notes in Computer Science 894, Springer Verlag, Berlin, 1995, 131-142.

20. O. Sýkora and 1 . Vrto, On the crossing number of hypercubes and cube connected cycles, BIT 33 (1993), 232-237.

21. A. T. White, L. W. Beineke, Topological graph theory, in: Selected Topics in Graph Theory, (L.W. Beineke R.J. Wilson, eds.), Academic Press, N.Y., 1978, 15-50.

22. K. Wada, K. Kawaguchi, H. Suzuki, Optimal bounds of the crossing number and the bisection width for generalized hypercube graphs, in: Proc. 16th Biennial Symposium on Communications, 1992, 323-326. 